\title{
Puerperal septic shock and necrotizing fasciitis caused by Staphylococcus caprae and Escherichia coli
}

\author{
Yu-Jin Koo \\ Department of Obstetrics and Gynecology, Yeungnam University College of Medicine, Daegu, Korea
}

\begin{abstract}
Puerperal sepsis is one of the leading causes of maternal morbidity and mortality worldwide. Postpartum pelvic infections can cause various complications, including wound infections and necrotizing fasciitis. Several microorganisms are known to cause such infections; however, no study has reported on Staphylococcus caprae, a coagulase-negative staphylococcus that is isolated frequently from animals and infrequently from human specimens, as a causative agent. Here, we report a rare case of septic shock complicated by necrotizing fasciitis after a cesarean section. This is the first report of a human isolate of $S$. caprae in association with puerperal sepsis and necrotizing fasciitis.
\end{abstract}

Keywords: Postpartum period; Puerperal infection; Septic shock

\section{INTRODUCTION}

The global maternal death rate is decreasing every year; however, it remains high in many countries. In 2015, the maternal mortality ratio was 216 per 100,000 live births worldwide and 11 per 100,000 live births in South Korea [1]. Although septic shock rarely occurs in pregnancy, infection is one of the most common direct causes of maternal death. Puerperal sepsis is estimated to cause the death of more than 75,000 pregnant women every year worldwide [2].

The common clinical features of sepsis include fever, rigors, diarrhea, vomiting, rash, and abdominal or pelvic tenderness. Sepsis is diagnosed clinically; however, thorough investigations, including relevant imaging studies, blood cultures, and any other tests based on the clinical suspicion of the origin of infection, should be performed for the diagnostic workup. The

Received: August 25, 2018, Revised: October 5, 2018

Accepted: October 10, 2018

Corresponding Author: Yu-Jin Koo, Department of Obstetrics and Gynecology, Yeungnam University College of Medicine,

170, Hyeonchung-ro, Nam-gu, Daegu 42415, Korea

Tel: +82-53-620-3433, Fax: +82-53-654-0676

E-mail: yujinkoo@yu.ac.kr intravenous administration of broad-spectrum antibiotics is the key treatment modality.

The most common organisms that cause puerperal sepsis are Escherichia coli, Staphylococci, and Streptococci. Particularly, infections caused by group A Streptococci are widely responsible for severe maternal sepsis and death. In addition, it is well known that staphylococcal toxic shock syndrome (TSS), caused by Staphylococcus aureus, is a life-threatening illness that is associated with necrotizing fasciitis. However, few case reports or case series have reported Staphylococcus caprae as a cause of human infection. Here, we report a rare case of necrotizing fasciitis after a cesarean section. This is the first report of a human isolate of $S$. caprae in association with puerperal sepsis and necrotizing fasciitis.

\section{CASE}

Patient: Female, 30 years old.

Presenting complaint: A sudden gush of clear fluid from the vagina.

Obstetric history: 1-0-0-1, a history of vaginal delivery at term, 1 year ago.

Copyright (C) 2018 Yeungnam University College of Medicine

This is an Open Access article distributed under the terms of the Creative Commons Attribution Non-Commercial License (http://creativecommons.org/licenses/by-nc/4.0/) which permits unrestricted non-commercial use, distribution, and reproduction in any medium, provided the original work is properly cited. 
History of presenting complaint: A woman with a singleton pregnancy at 26 weeks of gestation, visited a local clinic because of abdominal pain and vaginal leakage of amniotic fluid that occurred four hours prior to presentation. She was diagnosed with preterm labor and membrane rupture and was referred to our emergency department.

Past history: She conceived naturally and was followed up regularly at an obstetric clinic from an early gestational age. There were no specific findings in her antenatal course or in her medical or surgical history. She was taking oral folic acid and iron supplements.

Physical examination: At presentation, the patient showed the following: body mass index, $21.2 \mathrm{~kg} / \mathrm{m}^{2}$; blood pressure, $110 / 80 \mathrm{mmHg}$; pulse rate, 88 beats/min; and body temperature, $37.5^{\circ} \mathrm{C}$. On speculum examination, there was a large volume of clear fluid in the vagina, which was positive to the nitrazine test.

Laboratory findings: Blood testing showed increased levels of

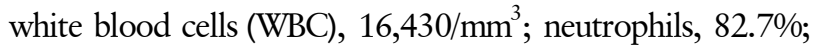
and C-reactive protein (CRP), $1.9 \mathrm{mg} / \mathrm{dL}$ (range, $<0.5 \mathrm{mg} / \mathrm{dL}$ ). Hemoglobin levels and platelet counts were normal. The results of the coagulation profile, liver function test, renal function test, and urine analysis were normal. Tests for congenital intrauterine infection and sexually transmitted diseases were not performed at patient presentation.

Imaging findings: Ultrasonography showed the fetus in a breech presentation, with an estimated fetal weight of $750 \mathrm{~g}$ (9th percentile) and an amniotic fluid index of $1.6 \mathrm{~cm}$. The cervical length was $1.8 \mathrm{~cm}$. On electronic fetal monitoring, the fetal heart rate was 148 beats/min with moderate variability, and there were uterine contractions every 2-3 min.

Management and clinical progress: Since she was hospitalized, absolute bed rest was recommended, and several medications, including dexamethasone, ritodrine, and ceftezole, were intravenously administered. Although a small amount of fluid leakage continued from her vagina, the abdominal pain improved after admission, and her body temperature remained normal at $36.9^{\circ} \mathrm{C}$.

Three days after hospital admission (at $26+3$ weeks of gestation), the patient complained of a headache with myalgia, and her body temperature was $38.2^{\circ} \mathrm{C}$. With a clinical diagnosis of chorioamnionitis, the patient underwent an emergency cesarean section. At surgery, there was no meconium in the amniotic fluid, but both the amniotic membrane and uterine endometrium were stained yellow. After delivery of the placenta, a swab culture from the endometrium was obtained, in which an extended-spectrum $\beta$-lactamase-producing $E$. coli was isolated. During surgery, no significant hemorrhage or surgical complication occurred.

Thirty minutes after the end of surgery, she experienced a sudden onset of massive vaginal bleeding. Evaluation showed the following: blood pressure, 90/40 $\mathrm{mmHg}$; pulse rate, 128 beats/min; and body temperature, $39.5^{\circ} \mathrm{C}$. Her mental state became drowsy, and a boggy uterus was palpable above the umbilicus. Since her central venous pressure was below $3 \mathrm{mmHg}$, a large volume of intravenous fluid was infused, and a transfusion was performed. The estimated blood loss was approximately $1,000 \mathrm{~mL}$, and the patient was suspected to have uterine atony, complicated by septic shock. We managed the patient with oxygen delivered via nasal cannula, intravenous administration of uterine tonics (methylergometrine and carbetocin), antibiotics (ceftriaxone and metronidazole), and antipyretics. Arterial blood gas analysis showed metabolic acidosis ( $\mathrm{pH}, 7.33 ; \mathrm{pCO}_{2}, 27 \mathrm{mmHg} ; \mathrm{pO}_{2}, 147 \mathrm{mmHg}$; base excess, $-10.2 \mathrm{mmol} / \mathrm{L} ; \mathrm{HCO}_{3}^{-}, 14.1 \mathrm{mmol} / \mathrm{L}$; and lactate, $1.8 \mathrm{mmol} / \mathrm{L}$ ). The blood test results, including increased levels of WBC $\left(33,510 / \mathrm{mm}^{3}\right), \mathrm{CRP}(15.4 \mathrm{mg} / \mathrm{dL})$, procalcitonin $(42.1 \mathrm{ng} / \mathrm{mL}$; range, $<0.5 \mathrm{ng} / \mathrm{mL})$, and D-dimer $(324 \mu \mathrm{g} / \mathrm{mL})$; decreased levels of hemoglobin $(7.1 \mathrm{~g} / \mathrm{dL})$, platelets $\left(76,000 / \mathrm{mm}^{3}\right)$, antithrombin III (48\%), and fibrinogen $(<80 \mathrm{mg} / \mathrm{dL})$; and prolonged times of prothrombin and activated partial thromboplastin, were consistent with infection and disseminated intravascular coagulation. Cultures from blood, urine, and sputum all yielded negative results. On transvaginal sonography, there was no significant finding in the uterus, and both the cervix and vagina were normal. After several minutes of uterine massage, uterine contractions were ensured, and vaginal bleeding was controlled. The patient was admitted to the intensive care unit (ICU), and treated with an intravenous administration of tranexamic acid, anti-thrombin III, and norepinephrine. Two days after surgery, the antibiotics were changed to a combination of ampicillin/sulbactam and metronidazole based on the assumption of sepsis caused by group A Streptococcus. However, because a swab culture of vaginal discharge yielded an extended-spectrum $\beta$-lactamase-producing $E$. coli, the antibiotics were changed to intravenous ertapenem $(1 \mathrm{~g}$, once a day) on the fifth day after surgery.

After treatment, her condition and laboratory findings im- 

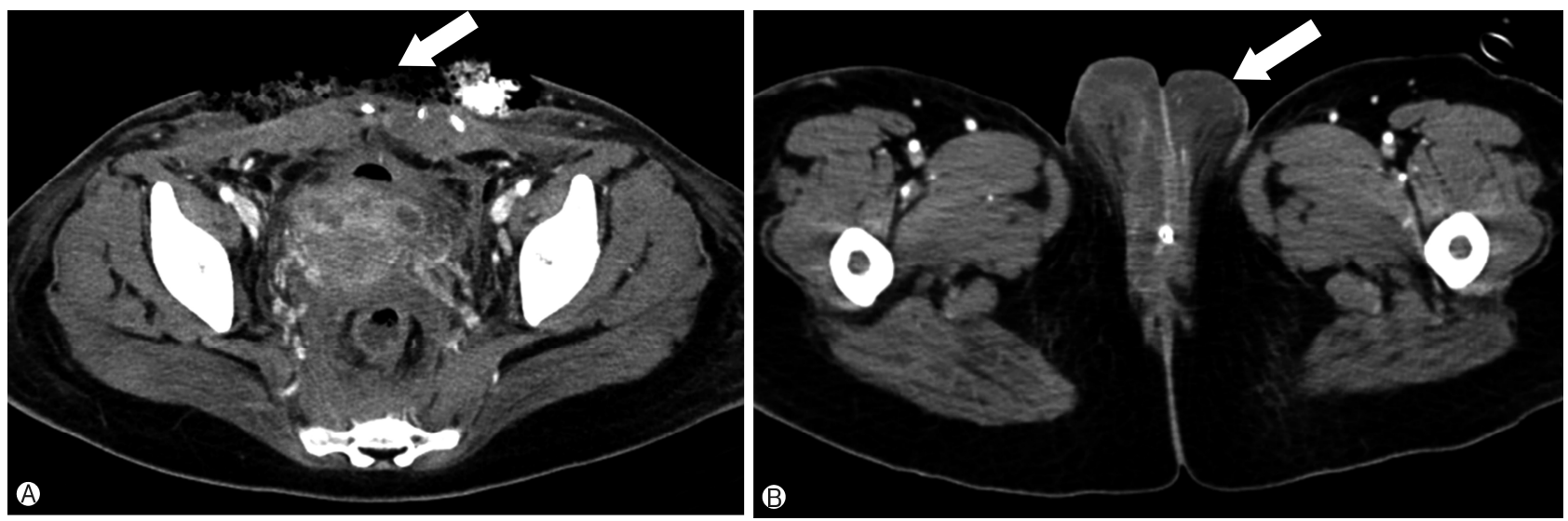

Fig. 1. Contrast-enhanced pelvic computed tomography images. (A) A negative pressure wound therapy dressing was placed after the cesarean scar was opened (arrow). (B) An axial image showing severe swelling and inflammatory infiltrates in the vulva (arrow).

proved, but fever, up to $39^{\circ} \mathrm{C}$ recurred, and both WBC $(18,160$ / $\left.\mathrm{mm}^{3}\right)$ and CRP $(30.3 \mathrm{mg} / \mathrm{dL})$ levels increased again on the seventh postoperative day. She presented tenderness in the lower abdomen and vulva. Exudate discharge from the cesarean scar was detected, and inflamed necrotic skin with severe edema, a color change to dark, and scaling were observed on her vulva. Because a clinical diagnosis of surgical site infection with necrotizing fasciitis caused by uterine infection was made, the cesarean fascia scar was opened. During surgical exploration, loculated fluid with necrotic tissue was observed, so debridement with copious irrigation using a Versajet was performed under local anesthesia. On postoperative computed tomography, there was no evidence of either intraabdominal abscess or pelvic thrombophlebitis (Fig. 1). A culture of the pus collected from the abdominal fascia yielded penicillin-sensitive Staphylococcus caprae. A combination of ertapenem and ampicillin/sulbactam was administered, and aggressive debridement with wound dressing was performed daily. A gradual return to the normal appearance of both the abdominal wound and vulva was observed. Other symptoms, including myalgia, diarrhea, diffuse skin rash, and pleural effusion progressively improved. Two weeks after surgical exploration, the abdominal wound was closed using a local advancement flap by plastic surgeons. The patient was discharged one week after the repair surgery, without evidence of wound complications.

At birth, the neonatal weight was $840 \mathrm{~g}$, and Apgar scores were 5 at $1 \mathrm{~min}$ and 7 at $5 \mathrm{~min}$. After being managed with a mechanical ventilator in the neonatal ICU, the neonate was discharged 3 months after birth and has been healthy during 1 year of follow-up.

\section{DISCUSSION}

In 2012, the Royal College of Obstetricians and Gynaecologists released the Green-top Guidelines regarding the diagnostic criteria and management recommendations for bacterial sepsis in pregnancy [3] and following pregnancy [4]. Based on these guidelines, the present case can be diagnosed as puerperal sepsis and is classified as a probable case of staphylococcal toxic shock. Multiple risk factors for puerperal infection have been reported in the literature, and the delivery mode of cesarean section is the single most powerful factor [2]. Other risk factors include vaginal discharge and prolonged spontaneous rupture of membranes, which corresponds with the features of the present case. Additional factors, such as obesity, diabetes, anemia, immunosuppressed condition, and a history of pelvic infection, are also associated with an increased risk of puerperal infection.

The most common organisms that cause puerperal sepsis are E. coli, Staphylococcus, and Streptococcus. Other organisms include Klebsiella, Clostridium sordellii, Mycoplasma, Chlamydia, and coliform bacteria [5]. In a prospective cohort study of 151 women who experienced puerperal sepsis, the authors found that the most common bacterial isolates were E. coli (30.6\%) and Klebsiella pneumoniae(15.3\%), followed by coagulase-negative staphylococci and $S$. aureus [6]. It has long been recognized that strains of $S$. aureus, which cause TSS, are capable of producing several toxins, such as TSS toxin- 1 (TSST-1) and other enterotoxins. However, little is known about $S$. caprae as a causative organism in human infections.

S. caprae is a coagulase-negative, Gram-positive coccus that 
is mainly isolated from animals and was first isolated from goat's milk in 1983 [7]. The incidence rate of $S$. caprae infection in humans is unclear. In a large study, staphylococcal species were genetically identified by DNA-DNA hybridization, and $10.7 \%$ of 1,230 staphylococcal strains were $S$. caprae, suggesting that this organism is widely distributed in human clinical specimens [8]. In contrast, in a study of patients with acute otitis externa, among the 107 coagulase-negative staphylococci recovered from clinical specimens, only one strain was $S$. caprae [9]. Whether $S$. caprae was the true cause of the infections in these published cases is unclear. In a study of goat specimens, three (16.5\%) out of 86 staphylococcal strains that produced TSST-1 were $S$. caprae [10]. In a few studies of human clinical specimens, $S$. caprae was isolated from patients with endocarditis, acute otitis externa, bone and joint infections, urinary tract infections, meningitis, and bacteremia, as well as from infants in neonatal ICUs. Most of these infections were hospital acquired, and a few were community acquired. The first human case of $S$. caprae meningitis, which was described in 2008, was associated with a nosocomial infection of an intraspinal device [11]. In addition, Vandenesch et al. [12] reported five cases of human infection with $S$. caprae and stated that this organism is a human pathogen responsible for severe clinical diseases both in the hospital and in the community.

In the present case, the diagnosis was based on a positive culture result and gross evidence of a necrotic wound during surgical debridement, from which $S$. caprae was isolated by culture. The culture result indicates that the secondary wound infection and necrotizing fasciitis were caused by $S$. caprae, following the ascending infection of the uterus from the vagina caused by $E$. coli. Thus, the patient likely acquired $S$. caprae during her hospital stay. However, the actual timing of the infection is unknown because all samples were obtained after the administration of antibiotics.

S. caprae is usually sensitive to most anti-staphylococcal antibiotics, including penicillin; however, some resistance to methicillin has been reported [13]. Furthermore, a recent study showed that $S$. caprae and methicillin-resistant $S$. aureus share phenotypic and genotypic characteristics, and are capable of persisting for a long time, particularly in an ICU setting [14]. Maternal sepsis can rapidly progress to septic shock, which has a mortality rate of $20-28 \%$ [15]. Therefore, knowledge of this infrequently isolated organism is essential, particularly in ICU settings, to promote early identification and appropriate management.

Early detection of sepsis is particularly challenging during pregnancy and the postpartum period due to its insidious onset. A delay in the diagnosis of septic shock is one of the factors predicting poor prognosis. The clinical information in the present case extends our knowledge of $S$. caprae, a rarely reported organism in humans with severe infection. To the best of our knowledge, this is the first report of necrotizing fasciitis caused by $S$. caprae complicated by puerperal septic shock.

\section{CONFLICT OF INTEREST}

No potential conflict of interest relevant to this article were reported.

\section{ORCID}

Yu-Jin Koo, https://orcid.org/0000-0002-5114-8000

\section{REFERENCES}

1. World Health Organization. World health statistics 2017: monitoring health for the SDGs [Internet]. Geneva: World Health Organization; 2017 [cited 2018 May 23]. http://www. who.int/gho/publications/world health_statistics/2017/en/

2. van Dillen J, Zwart J, Schutte J, van Roosmalen J. Maternal sepsis: epidemiology, etiology and outcome. Curr Opin Infect Dis 2010;23:249-54.

3. Royal College of Obstetricians and Gynaecologists. Bacterial sepsis following pregnancy [Internet]. Green-top guideline no. 64a. 2012 [cited 2018 May 23]. https://www.rcog.org.uk/ globalassets/documents/guidelines/gtg_64a.pdf

4. Royal College of Obstetricians and Gynaecologists. Bacterial sepsis following pregnancy [Internet]. Green-top guideline no. 64b. 2012 [cited 2018 May 23]. https://www.rcog.org.uk/ globalassets/documents/guidelines/gtg_64b.pdf

5. Aronoff DM, Mulla ZD. Postpartum invasive group A streptococcal disease in the modern era. Infect Dis Obstet Gynecol 2008;2008:796892.

6. Majangara R, Gidiri MF, Chirenje ZM. Microbiology and clinical outcomes of puerperal sepsis: a prospective cohort study. J Obstet Gynaecol 2018;38:635-41.

7. Devriese LA, Poutrel B, Kilpper-Bälz R, Schleifer KH. Staphylococcus gallinarum and Staphylococcus caprae, two new species from animals. Int J Syst Evol Microbiol 1983;33:480-6.

8. Kawamura Y, Hou XG, Sultana F, Hirose K, Miyake M, Shu $\mathrm{SE}$, et al. Distribution of Staphylococcus species among human clinical specimens and emended description of Staphylococcus 
caprae. J Clin Microbiol 1998;36:2038-42.

9. Roland PS, Stroman DW. Microbiology of acute otitis externa. Laryngoscope 2002;112:1166-77.

10. Valle J, Vadillo S, Piriz S, Gomez-Lucia E. Toxic shock syndrome toxin 1 (TSST-1) production by staphylococci isolated from goats and presence of specific antibodies to TSST- 1 in serum and milk. Appl Environ Microbiol 1991;57:889-91.

11. Benedetti P, Pellizzer G, Furlan F, Nicolin R, Rassu M, Sefton A. Staphylococcus caprae meningitis following intraspinal device infection. J Med Microbiol 2008;57:904-6.

12. Vandenesch F, Eykyn SJ, Bes M, Meugnier H, Fleurette J,
Etienne J. Identification and ribotypes of Staphylococcus caprae isolates isolated as human pathogens and from goat milk. J Clin Microbiol 1995;33:888-92.

13. Kini GD, Parris AR, Tang JS. A rare presentation of sepsis from Staphylococcus caprae. Open Microbiol J 2009;3:67-8.

14. Shuttleworth R, Behme RJ, McNabb A, Colby WD. Human isolates of Staphylococcus caprae: association with bone and joint infections. J Clin Microbiol 1997;35:2537-41.

15. Barton JR, Sibai BM. Severe sepsis and septic shock in pregnancy. Obstet Gynecol 2012;120:689-706. 